\title{
A Phase li Study Of The Combination Of Oxaliplatin, Capecitabine, And Trastuzumab And Chemo- radiotherapy In The Adjuvant Setting In Operated Patients With Her2+ Gastric Or Gastroesophageal Junction Cancer (Toxag Study), A Turkish Oncology Group Study
}

Huseyin Abali ( $\sim$ dr.huseyinabali@yahoo.com )

Acibadem Adana Hastanesi https://orcid.org/0000-0002-5272-8672

Suayib Yalcin

Hacettepe Universitesi Onkoloji Enstitusu

Huseyin Cem Onal

Baskent Universitesi Tip Fakultesi

Faysal Dane

Marmara Universitesi Tip Fakultesi

Berna Oksuzoglu

Dr Abdurrahman Yurtaslan Onkoloji Egitim ve Arastirma Hastanesi

Nuriye Ozdemir

Gazi Universitesi Tip Fakultesi

Huseyin Mertsoylu

Baskent Universitesi Tip Fakultesi

Mehmet Artac

Necmettin Erbakan Universitesi Meram Tip Fakultesi

Celaletdin Camci

Ethica Incirli Hastanesi

Bulent Karabulut

Ege Universitesi Tip Fakultesi

Fatma Bugdayci Basal

Dr Abdurrahman Yurtaslan Onkoloji Egitim ve Arastirma Hastanesi

Burcin Budakoglu

Medical Park Ankara Hastanesi

Mehmet Ali Sendur

Ankara Yildirim Beyazit Universitesi Tip Fakultesi

Burce Goktaş 
Roche Mustahzarlari Sanayi Anonim Sirketi

\section{Fatih Ozdener}

Bahcesehir Universitesi

\section{Arzu Baygul}

Beykent Universitesi

\section{Research article}

Keywords: Gastric, gastroesophageal, HER-2, trastuzumab, gastrectomy, D2 dissection

Posted Date: March 5th, 2020

DOl: https://doi.org/10.21203/rs.3.rs-16111/v1

License: (c) (i) This work is licensed under a Creative Commons Attribution 4.0 International License. Read Full License 


\section{Abstract}

\section{Background}

Trastuzumab prolonged the overall survival in patients with advanced gastric cancer with HER2 overexpression in combination with chemotherapy. In this phase II open-label prospective study, the tolerability and safety of trastuzumab with chemotherapy, and chemoradiotherapy for curatively resected patients with HER2 + gastric carcinoma was investigated.

\section{Methods}

The patients with HER2-positive gastric, or gastroesophageal junction adenocarcinoma, after gastrectomy plus D2 dissection were included. They received 3 cycles of oxaliplatin $(100 \mathrm{mg} / \mathrm{m} 2 \mathrm{IV}$ day 1) plus capecitabine ( $850 \mathrm{mg} / \mathrm{m} 2$ PO days $1-14)$, trastuzumab (8 mg/kg IV day 1 in cycle $1,6 \mathrm{mg} / \mathrm{kg}$ thereafter) every 21 days, followed by chemoradiotherapy. Trastuzumab was given for 1 year.

\section{Results}

Of the 212 patients screened, 35 were eligible, and 34 were treated. The median age was 56 years (Minmax: $35-75)$, male patients constituted $73.5 \%(n=25)$, and $33(97.1 \%)$ had gastric adenocarcinoma. $R 0$ resection was performed in $30(88.2 \%)$. The majority $(26,61.7 \%)$ were in stage III disease. Most of the AEs were grade I/II, the most frequent grade III side effects were nausea $(3,8.8 \%)$, vomiting $(3,8.8 \%)$, diarrhoea $(2,5.9 \%)$ and weight loss $(\mathrm{N}=2,5.9 \%)$. Two patients died during the first 3 cycles of chemotherapy and chemoradiotherapy; 1 secondary to pulmonary thrombo-embolism, and the other due to cerebral ischemia.

After excluding 2 with early progression and 1 consent withdrawal, of the remaining 31 patients, 28 (90.3\%) were able to complete the chemotherapy and chemoradiotherapy part of the trial. After the 25 months follow up period, 21 patients (61.8\%) were alive. Overall survival at 12 and 24 months was $75.0 \%$ and $65.7 \%$, while disease-free survival at 12 and 24 months was $65.7 \%$ and $55.0 \%$, respectively.

\section{Conclusions}

Trastuzumab in combination with capecitabine, oxaliplatin and radiotherapy as the adjuvant therapy for gastric or gastroesophageal junction adenocarcinoma was considered safe and tolerable. The frequency of HER2 overexpression in curatively resected patients is comparable to that in patients with metastatic disease.

\section{Background}

Gastric cancer is the $4^{\text {th }}$ most frequently seen and the 2 nd most frequently observed cause of cancerrelated deaths in the world [1]. Gastric and gastroesophageal junction adenocarcinoma both show high mortality rates if not curatively treated at an early stage of the disease [2]. Adequate surgical resection is 
the only potentially curative treatment modality in early stages, but is not without recurrence and unfortunately mortality [2].

Human epidermal growth factor receptor (HER2) is a member of the human epidermal growth factor receptors that regulates cell proliferation, differentiation, and survival [3]. Observed in $12-20 \%$ of gastric adenocarcinomas in patients with advanced stages of gastric cancer, the HER2 overexpression is a validated predictive biomarker and targeting it is associated with prolonged overall survival (OS) and progression free survival (PFS) in the same set of patients [4, 5]. Trastuzumab, an anti-HER2 monoclonal antibody, was first developed for the treatment of HER2-positive metastatic breast cancer [4]. Currently in breast cancer, chemotherapy plus trastuzumab plus/minus pertuzumab is the standard therapy in cases with HER2 overexpression in both adjuvant and metastatic setting [6, 7]. In gastric cancer, in the pivotal TOGA trial where patients were randomized in two arms with chemotherapy with or without trastuzumab, the median overall survival of patients receiving chemotherapy and trastuzumab was improved from 11.8 months 16.0 months in HER2 IHC 2 positive / FISH positive or IHC 3 positive advanced gastric cancer patients [5]. It was a major step forward after many failures or at best modest successes of various combinations of chemotherapeutics. The ToGA trial changed the standard of care to the combination of trastuzumab, cisplatin and fluoropyrimidine as the first line treatment of advanced HER2-positive gastric, and gastroesophageal junction adenocarcinomas [2].

At this time in operable, non-metastatic stages of gastric and esophagogastric junction tumours, there are no universally accepted neoadjuvant or adjuvant treatment strategies in addition to curative surgery. In Japan and the Far East only adjuvant chemotherapy [8], in Europe perioperative chemotherapy, and in North America adjuvant chemoradiotherapy have been adopted in addition to gastrectomy plus D2 lymphadenectomy [2, 9-11].

The improvement in overall survival rates of patients with HER2 positive advanced gastric cancer when trastuzumab was used in combination with chemotherapy in the ToGA trial [5] led us to consider its potential added benefit in the adjuvant setting in advanced HER2 positive gastric cancer. Therefore, we designed this study to investigate the safety and tolerability of incorporating trastuzumab into adjuvant chemotherapy and chemoradiotherapy of curatively resected patients with HER2 over-expressing gastric or gastroesophageal junction carcinoma.

\section{Methods}

\section{Study Design and participants}

This was a single-arm, open-label, non-comparative, multicentre, national phase II study, designed and initiated by the Turkish Oncology Group. It was conducted in medical and radiation oncology departments of 9 hospitals in 5 different cities in Turkey. All eligible female or male patients between 18 and 75 years of age with curatively resected (partial or total gastrectomy, R0 or R1 resection) HER2positive, gastric or gastroesophageal junction adenocarcinoma were enrolled into the study by the 
treating medical oncologist. Like in the ToGA trial [5] volunteers were eligible if their tumour samples were assessed as 3 positive on immunohistochemistry, or if they were FISH positive with HER2:CEP17 ratio $\geq 2$, confirmed by the central laboratory. Other inclusion criteria were: patients with stage IB (T1N1M0) to Stage IIIC (both stages inclusive) according to AJCC $7^{\text {th }}$ edition, 2010 [12]; ECOG performance status $\leq 2$ at screening, and left ventricular ejection fraction $\geq 55 \%$, acceptable levels of liver and renal functions. Key exclusion criteria were: any malignancy in the last 5 years (except for squamous cell carcinoma of the skin), congestive heart failure, transmural myocardial infarction, uncontrolled hypertension (systolic blood pressure $>180 \mathrm{mmHg}$, or diastolic blood pressure $>100 \mathrm{mmHg}$ ), angina pectoris requiring medical intervention, clinically significant valvular heart diseases, high-risk arrhythmias, and abnormal laboratory values. In addition, patients with any known disorders which may cause upper gastrointestinal tract dysfunction (excluding the surgical intervention performed for gastric or gastroesophageal junction carcinoma), known malabsorption syndrome, or active gastrointestinal bleeding were excluded from this study.

The central ethics committee approval and approvals from the ethics committee from each participating institution were obtained prior to the study initiation. Investigators provided information to the patients about the clinical study and all patients signed a written informed consent before initiation of any studyrelated procedures. This trial was conducted in accordance with the Helsinki declaration. The trial was registered in clinicaltrials.gov with the identifier NCT01748773.

\section{Procedures}

After curative surgery, 3 cycles of oxaliplatin $\left(100 \mathrm{mg} / \mathrm{m}^{2}\right.$ intravenously on day 1$)$, capecitabine (850 $\mathrm{mg} / \mathrm{m}^{2}$ orally twice daily on days 1 through 14 ) combination chemotherapy (CAPOX) plus trastuzumab (at a loading dose of $8 \mathrm{mg} / \mathrm{kg}$ intravenously in the $1^{\text {st }}$ cycle, followed by $6 \mathrm{mg} / \mathrm{kg}$ thereafter) every 21 days followed by chemoradiotherapy with oral capecitabine were administered. Trastuzumab was continued during chemoradiotherapy and completed to 1 year (Figure 1). After these first 3 cycles, the patients were assessed for radiotherapy and eligible patients started to receive chemoradiotherapy on the day 1 of the $4^{\text {th }}$ cycle and continued for a total of 5 weeks with 5 treatment sessions in each week. The prescribed dose was $45 \mathrm{~Gy}$ delivered with a fraction size of $1.8 \mathrm{~Gy}$. All of the plans were normalized to deliver the prescribed dose to $>95 \%$ of the planning target volume (PTV) and to $99 \%$ of the clinical target volume (CTV), while the PTV was not permitted to receive more than $115 \%$. During the chemoradiotherapy, capecitabine was administered orally during radiation therapy on weekdays (not during weekends); trastuzumab was continued on the $1^{\text {st }}$ and $22^{\text {nd }}$ days.

Imaging work up as thoracic, abdomen and pelvic computerized tomography (CT) one week prior to chemoradiation onset part were performed. Ejection fraction was followed by echocardiographic every 3 months for trastuzumab cardiac toxicity. The subjects were followed for relapse after chemoradiotherapy every 3 months up to 1 year with the protocol, and according to treating physician's discretion thereafter. One month after completing the $12^{\text {th }}$ cycle of trastuzumab, the patients were invited to a follow-up visit 
(safety follow-up visit) at month 13 after the start of the treatment to collect safety information. Twelve months after the treatment period, patients were contacted to assess disease-free survival (DFS) and overall survival (OS). Adverse events were graded by Common Terminology Criteria for Adverse Events (CTCAE), version 4.03.

\section{Outcome measurements}

The primary endpoint was safety and tolerability of the first 3 cycles for chemotherapy and the chemoradiotherapy in combination with trastuzumab in the adjuvant setting. The patients completing chemoradiotherapy with 2 dose reductions were considered tolerable.

The secondary endpoints were DFS and OS. Time from the first oxaliplatin, capecitabine and trastuzumab adjuvant therapy to relapse and/or progressive disease (PD) and/or death, whichever occurred first, was determined as DFS. In addition, time from the first oxaliplatin and capecitabine chemotherapy and trastuzumab adjuvant therapy to the time of death due to any cause was determined as OS.

\section{Statistical Analysis}

\section{Sample Size Calculation}

The current prevalence of gastric or gastroesophageal junction adenocarcinoma was estimated to be $0 \cdot 8-0 \cdot 1 \%[1]$. The sample size calculation was based on this prevalence rate, and other phase $\mathrm{I} / \mathrm{II}$ studies of similar design which were performed with oxaliplatin and capecitabine in gastric cancer treatment. In these studies, the sample sizes were 38 and 50 (standard deviation not specified). Therefore, an effect size with $a=0.05$ (two-sided $t$ value) and $d=0.433$ was calculated, our target sample size was determined as 40 patients.

In the landmark study of INT0116 where the patients received chemoradiotherapy after curative resection, $64.0 \%$ of the patients were able to complete the whole treatment plan [9]. Our primary endpoint was defined as the safety and tolerability of our treatment protocol after curative resection. We presumed that our study would be considered positive if $80 \%$ of the patients could receive 3 cycles of trastuzumab plus chemotherapy followed by chemoradiation with 2 dose modifications.

\section{Interim Analysis}

In order to avoid unnecessary patient recruitment, an interim analysis after $20^{\text {th }}$ patient finished the chemoradiotherapy was planned. By pre-defining an $\alpha=0.10$ and $\beta=0.64$, fixed comparisons would be carried out by Fisher's Exact test. If the predefined a value is significantly better than the tolerability 
percentage of $64.0 \%$ observed in INT0116, we would continue to recruit more patients up to 40 , which was ultimately the responsibility of the Independent Data and Safety Monitoring Committee, which was presented previously [13].

For survival analysis, non-parametric estimations of survival distribution function were calculated according to Kaplan Meier and the rest according to descriptive methods. All patients receiving the study medication were included in all analyses since the aim of this clinical study was to investigate the tolerability, safety and DFS and OS rates of oxaliplatin-capecitabine- trastuzumab combination. MedCalc Statistical Software version 12.7.7 (MedCalc Software bvba, Ostend, Belgium; 2013) was used for all the statistical analyses.

\section{Reporting of the trial}

This study has been written in line with Consolidated Standards of Reporting Trials (CONSORT) Statement. Since it is not a randomized trial, CONSORT extension for Pilot and Feasibility Trials Checklist has been filled in.

\section{Role of the funding source}

Roche Pharmaceuticals Inc. Turkey sponsored the TOXAG study. It was designed by the Turkish Oncology Group investigators in collaboration with the sponsor. The investigators and the sponsor collaboratively performed data collection, interpretation and manuscript preparation. The corresponding author had access to the study data and the final version of the manuscript was reviewed by all the authors.

\section{Results}

From 21 November 2012 to 31 December 2015, a total of 212 patients were screened. The first patient visit was on 29 January 2013, and last patient's follow-up ended on 01 July 2016. The study was terminated because of poor-accrual after a total of 35 patients with HER2 positive disease $(16.5 \%$ of the screened patients) were included, one patient was ineligible for analysis because of faulty inclusion (inconsistent staging). The patient disposition is presented in Figure 2.

Of 34 eligible patients, the median age was 56 (minimum-maximum (35-75) years and male patients constituted $73.5 \%(n=25)$ and 33 patients $(97.1 \%)$ had gastric adenocarcinoma.

Gastrectomy with R0 resection was performed in 30 (88.2\%) and 33 patients had D2 lymph node dissection. The most common stage was stage IIIC (41.1\%) followed by stage IIIA (20.6\%), hence stage III constituted $61.7 \%$ of the recruited patients. According to the immunohistochemistry (IHC) results, 19 patients (55.9\%) were detected as ICH 2 positive /FISH positive and 15 patients $(44.1 \%)$ were ICH 3 positive / FISH positive. At the baseline visit, the ECOG performance status was I for 20 patients (58.8\%) and ' 0 ' for 13 patients (38.2\%). The key clinical features are summarized in Table 1. 
A total of 288 adverse events (AEs) were observed during the study, with $12(4.2 \%)$ serious adverse events (SAEs) in 8 patients $(23.5 \%)$. In total, 24 AEs were related to trastuzumab $(12.4 \%)$. Most of the AEs were within the range of grade $\mathrm{I} / \mathrm{II}$, the most frequent grade III side effects were nausea $(n=3,8.8 \%)$ and vomiting $(n=3,8.8 \%)$ patients, followed by diarrhoea $(n=2,5.9 \%)$ and weight loss $(n=2,5.9 \%)$. The toxicities experienced is summarized in Table 2. Additionally, trastuzumab and capecitabine treatments were discontinued permanently in 1 patient secondary to AE. There were 6 dose reductions ( $1 \mathrm{~T}, 2$ for 0 and 3 for $\mathrm{C}$ ). $\mathrm{T}$ was stopped in 1 patient; $\mathrm{C}$ was interrupted 11 times (mostly during radiotherapy) and stopped in 1 patient.

Of the study population, 2 patients, unfortunately, died during the first 3 cycles of chemotherapy and chemoradiotherapy; 1 secondary to pulmonary thrombo-embolism, and the other had to cerebral ischemia (thought to be due to Behçet's disease as a major contributing factor). No death was considered directly related to treatment by the investigators.

The patients completing 3 cycles of $\mathrm{O}, \mathrm{C}, \mathrm{T}$, and chemoradiotherapy were considered as tolerable patients by definition of tolerability in the design of the study, taking the tolerability rate of the similarly designed landmark INT 01-16 study. During this part of the treatment, 1 patient withdrew her/his consent before chemoradiotherapy and 2 patient dropped-out due to disease progression, therefore, these patients $(n=3)$ were excluded from tolerability analysis, leaving 31 patients for tolerability analysis. The consent withdrawal was not due to toxicity, but the patient was convinced by the operating surgeon who objected to chemoradiation, stating that there is no need for chemoradiotherapy. Therefore, tolerability analysis was carried out in 31 patients, 28 (90.3\%) were tolerable. Even if we do the calculation in the intent-totreat population of 34 patients, the tolerability rate was $82.4 \%$. One out of 3 intolerable patients had Behçet's disease as underlying disease, 1 patient was older than 75 years old the time of screening and 1 patient had an unresolved medical history of left ventricular dysfunction.

At the end of the study, a total of 21 patients $(61.8 \%)$ were alive $(n=21)$ at the end of 25 months followup period. The DFS@1 year and DFS@2 years were 65.7\% and 55.0\%, respectively (Figure 3).The 0S@ 1 year and OS@2 year were 75.0\% and 58.0\%, respectively (Figure 4). No deaths during therapy were reported as directly related to the treatment protocol. Since the survival rate has not fallen below $50 \%$ during the study, the median survival time could not be calculated.

\section{Discussion}

In this phase 2 study, safety and tolerability of oxaliplatin-capecitabine-trastuzumab combination and chemoradiotherapy in patients with curatively resected gastric or gastroesophageal junction adenocarcinoma were evaluated. To our best of knowledge, this is the first study evaluating trastuzumab in the adjuvant setting in gastric cancer.

In this study, we found that oxaliplatin-capecitabine-trastuzumab combination with chemoradiotherapy was better tolerated than the land-mark trial INT0116 study, where the completion rate adjuvant chemoradiotherapy was only $64.4 \%$ [9]. Generally, toxicities were in the order of grade I and II, grade III 
and worse toxicities were rare. Much of the toxicities were observed during the chemoradiation part. Compared to the INT0116 trial where grade III-IV haematological toxicities and gastrointestinal toxicities were seen in $54.0 \%$ and $33.0 \%$ of the patients respectively, the patients in our trial experienced fewer side effects of this kind. In the recently published trials, where chemoradiation is part of the treatment, toxicities were comparable to our trial [14-16]. Unfortunately, 2 patients died during treatment, which were not directly related to study medication. One of them had Behçet's disease, where thrombosis is a very-well-known-phenomenon. The other patient died of pulmonary embolism. The patient withdrawing the consent was not having significant toxicity, but the operating surgeon was against chemoradiation, she followed the surgeon's advice.

Maximum duration of follow-up was 25 months and by the end of this period, $61.8 \%$ of the patients were alive. The secondary endpoints were DFS and OS. The DFS@1 year and DFS@2 years were 65.7\% and $55.0 \%$, respectively. The OS@1 year 75.0\% and OS@2 year 58\% were similar to those observed in the previous trials $[9,14,16]$. Given the added benefit coming from trastuzumab in the ToGA trial, one might naturally expect that the survival results must have been better in our trial. In our opinion, the most probable reason for inferior than expected survival rates was the very advanced stage of patients in our trial. There were 14 patients (41.1\%) with stage IIIC where patients have beyond 15 lymph nodes involved by the disease. This may be either selection bias of the researchers who recruited rather advanced stage patients, or represent the actual stage distribution of the patients in Turkey $[17,18]$. The other explanation might be the unexpected early mortalities in our trial. Both were secondary to embolic events underlying the importance of thromboprophylaxis in patients with cancer.

One important find our study provides is the incidence of HER2 positivity in operable gastric and gastroesophageal cancer: It was positive in $16.5 \%$ (35 out of 212 patients screened), which is more, or less what is seen in advanced stages [5]. The other novelty it brings is that chemoradiation part can be applied after 3 cycles of adjuvant systemic treatment, the rationale behind which was the observation that majority of patients could not tolerate chemotherapy after chemoradiation. This approach may also give an opportunity to avoid unnecessary chemoradiotherapy in early-progressing patients with highly aggressive disease.

The major limitations of our study were its small sample size, early closure due to poor accrual, and 2 unexpected deaths. The other possible source of criticism may be the doses of capecitabine and oxaliplatin $(850 \mathrm{mg} / \mathrm{m} 2$ BID and $100 \mathrm{mg} / \mathrm{m} 2$, respectively. This issue was discussed in Turkish Oncology Group Protocol development meeting, and the majority found the doses of $1000 \mathrm{mg} / \mathrm{m} 2$ BID for capecitabine and $130 \mathrm{mg} / \mathrm{m} 2$ for oxaliplatin difficult to administer, needing dose reduction in majority of patients. The need for dose reduction in $90 \%$ of patients in CLASSIC trial support our dose preference, too [8].

There is an ongoing randomized phase II German study called PETRARCA Trial where patients with HER2positive locally-advanced gastric and gastroesophageal junction adenocarcinoma are randomized to 4 pre-operative 2-weekly cycles of FLOT (Docetaxel; Oxaliplatin; Leucovorin; 5-FU) followed by surgery and 
4 additional cycles of FLOT (arm A); or the same therapy in combination with trastuzumab and pertuzumab. If phase II part is positive, the phase III will be started (clinicaltrials.gov NCT identifier 2581462)[19]. In our opinion, the incremental benefit from pertuzumab may be questionable given the negative trial in metastatic setting [20]. The same group had published the single arm HER-FLOT trial perioperative trial with the same treatment schema, but without pertuzumab and had showed that there was no safety signal [19].

\section{Conclusion}

The TOXAG Study is the first trial on trastuzumab in combination with chemotherapy and chemoradiation and its results suggests that trastuzumab can be incorporated into adjuvant treatment of patients with HER2+ curatively resected gastric or gastroesophageal cancer. It also confirms that frequency of HER2 overexpression in curatively resected patients is comparable to that in patients with disease. We believe that a phase III trial with trastuzumab in perioperative or adjuvant setting treatment for such patients is warranted.

\section{Abbreviations}

HER2: Human epidermal growth factor receptor 2

OS: Overall Survival

PFS: Progression-free Survival

AJCC: American Joint Committee on Cancer

ECOG: Eastern Cooperative Oncology Group

PTV: Planning target volume

CTV: Clinical target volume

CT: Computerized tomography

CTCAE: Common Terminology Criteria for Adverse Events

PD: Progressive disease

IHC: Immunohistochemistry

AE: Adverse event

SAE: Serious adverse event

T: Trastuzumab 

O: Oxaliplatin
C: Capecitabine

\section{Declarations}

Ethics approval and consent to participate

The TOXAG Study was approved by Cukurova University Clinical Trials Ethics Committee (Date: 16 Feb 2012, Decision No: 6) and by Turkish Republic Ministry of Health Central Ethics Committee (Date:

09.10.2013, Number: 688784). Approval from Institutional Review of Board of each participating centre were obtained prior to the study initiation. Investigators provided information to the patients about the clinical study and all patients signed a written informed consent before initiation of any study-related procedures.

\section{Consent for publication}

Not applicable.

\section{Availability of data and materials}

The datasets used and/or analysed during the current study are available from the corresponding author and Roche Pharmaceuticals on a reasonable request.

\section{Competing interests}

Huseyin Abali: Honorarium/speaker, advisory support, research grant

Suayib Yalcin: Honorarium/speaker, advisory support, research grant

Huseyin Cem Onal: Honorarium/speaker, research grant

Faysal Dane: Honorarium/speaker, advisory support, research grant

Berna Oksuzoglu: Honorarium, congress support

Nuriye Ozdemir: No conflict of interest (for the last 2 years no congress support or honorarium)

Huseyin Mertsoylu: Travel grant, congress support.

Mehmet Artaç: Honorarium, congress support

Celaletdin Camcl: No conflict of interest (for the last 2 years no congress support or honorarium)

Bulent Karabulut: Honorarium, congress support, international clinical trials 
Fatma Bugdayci Basal: No conflict of interest (for the last 2 years no congress support or honorarium)

Burcin Budakoglu: Honorarium/speaker, advisory board, congress support

Mehmet Ali Nahit Sendur: Honorarium, congress support, international clinical trials

Burce Goktas: Current Roche employee

Fatih Ozdener: Ex-Roche employee (left the company in 2016)

Arzu Baygul: No conflict of interest

\section{Funding}

The study was fully sponsored by Roche Pharmaceuticals Inc. Turkey

\section{Authors' contributions}

$\mathrm{HA}$ and $\mathrm{HO}$ created study concepts. $\mathrm{HA}, \mathrm{SY}, \mathrm{HHO}, \mathrm{BK}$, and FO developed the study design. FD, BO, NO, HM, $\mathrm{MA}, \mathrm{CC}, \mathrm{BB}$, and FB carried out treatment, recruitment and data acquisition.

$H A, B G$ and $A B$ did data analysis and interpretation. The statistical analysis was performed by $A B$, and HA and MS the manuscript preparation. All authors read and approved the manuscript.

\section{Acknowledgments}

The authors would like to thank the patients, their families and all the investigators who participated in the study, the clinical operations team (Turkan Duman, Dr. Kasim Ustundag, IQVIA), data management team (Dr. Oguz Akbas and Murat Ozdemir), and Central Laboratory Targos Molecular Pathology, Germany.

\section{References}

1. International Agency for Research on Cancer. Cancer Fact Sheets: Stomach Cancer. World Health Organization. 2012. http://gco.iarc.fr/today. Accessed 12 Jan 2019.

2. E. C. Smyth, M. Verheij, W. Allum, D. Cunningham, A. Cervantes DA. Gastric cancer: ESMO-ESSOESTRO clinical practice guidelines for diagnosis, treatment and follow-up. Ann Oncol. 2016;27 Suppl 5:v38-49.

3. Akiyama T, Sudo C, Ogawara H, Toyoshima K YT. The product of the human c-erbB-2 gene: a 185kilodalton glycoprotein with tyrosine kinase activity. Science (80-). 1986;232:1644-6. http://science.sciencemag.org/content/sci/232/4758/1644.full.pdf.

4. Boku N. HER2-positive gastric cancer. Gastric Cancer. 2014;17:1-12. 
5. Bang Y-J, Van Cutsem E, Feyereislova A, Chung HC, Shen L, Sawaki A, et al. Trastuzumab in combination with chemotherapy versus chemotherapy alone for treatment of HER2-positive advanced gastric or gastro-oesophageal junction cancer (ToGA): a phase 3, open-label, randomised controlled trial. Lancet. 2010;376:687-97. doi:10.1016/S0140-6736(10)61121-X.

6. Baselga J, Swain SM. CLEOPATRA: A phase III evaluation of pertuzumab and trastuzumab for HER2positive metastatic breast cancer. Clin Breast Cancer. 2010;10:489-91.

7. Senkus E, Kyriakides S, Ohno S, Penault-Llorca F, Poortmans P, Rutgers E, et al. Primary breast cancer: ESMO Clinical Practice Guidelines for diagnosis, treatment and follow-up. Ann Oncol. 2015;26 Suppl 5:v8-30. doi:10.1093/annonc/mdv298.

8. Bang Y-J, Kim Y-W, Yang H-K, Chung HC, Park Y-K, Lee KH, et al. Adjuvant capecitabine and oxaliplatin for gastric cancer after D2 gastrectomy (CLASSIC): a phase 3 open-label, randomised controlled trial. Lancet. 2012;379:315-21. doi:10.1016/S0140-6736(11)61873-4.

9. Macdonald JS, Smalley SR, Benedetti J, Hundahl SA, Estes NC, Stemmermann GN, et al. Chemoradiotherapy after Surgery Compared with Surgery Alone for Adenocarcinoma of the Stomach or Gastroesophageal Junction. N Engl J Med. 2001;345:725-30. doi:10.1056/NEJMoa010187.

10. Cunningham D, Allum WH, Stenning SP, Thompson JN, Van de Velde CJH, Nicolson M, et al. Perioperative chemotherapy versus surgery alone for resectable gastroesophageal cancer. $\mathrm{N}$ Engl $\mathrm{J}$ Med. 2006;355:11-20. doi:10.1056/NEJMoa055531.

11. Sasako M, Sakuramoto S, Katai H, Kinoshita T, Furukawa H, Yamaguchi T, et al. Five-year outcomes of a randomized phase III trial comparing adjuvant chemotherapy with $S-1$ versus surgery alone in stage II or III gastric cancer. J Clin Oncol. 2011;29:4387-93.

12. Edge SB, Compton CC. The american joint committee on cancer: The 7th edition of the AJCC cancer staging manual and the future of TNM. In: Edge SB, Byrd DR, Compton CC, Fritz AC, Greene FL, Trotti A, editors. AJCC Cancer Staging Manual. Seventh Ed. Chicago: Springer; 2010. p. 117-26.

13. Abali H, Yalcin S, Onal HC, Dane F, Oksuzoglu B, Ozdemir N, et al. A study of the combination of oxaliplatin, capecitabine, and herceptin (trastuzumab) and chemoradiotherapy in the adjuvant setting in operated patients with HER2+ gastric or gastro-esophageal junction cancer (TOXAG study). J Clin Oncol. 2016;34 Suppl 4:TPS182. doi:10.1200/jco.2016.34.4_suppl.tps182.

14. Fuchs CS, Niedzwiecki D, Mamon HJ, Tepper JE, Ye X, Swanson RS, et al. Adjuvant Chemoradiotherapy With Epirubicin, Cisplatin, and Fluorouracil Compared With Adjuvant Chemoradiotherapy With Fluorouracil and Leucovorin After Curative Resection of Gastric Cancer: Results From CALGB 80101 (Alliance). J Clin Oncol. 2017;35:3671-7. doi:10.1200/JC0.2017.74.2130.

15. Leong T, Smithers BM, Michael M, Gebski V, Boussioutas A, Miller D, et al. TOPGEAR: a randomised phase III trial of perioperative ECF chemotherapy versus preoperative chemoradiation plus perioperative ECF chemotherapy for resectable gastric cancer (an international, intergroup trial of the AGITG/TROG/EORTC/NCIC CTG). BMC Cancer. 2015;15:532. doi:10.1186/s12885-015-1529-x. 
16. Cats A, Jansen EPM, van Grieken NCT, Sikorska K, Lind P, Nordsmark M, et al. Chemotherapy versus chemoradiotherapy after surgery and preoperative chemotherapy for resectable gastric cancer (CRITICS): an international, open-label, randomised phase 3 trial. Lancet Oncol. 2018;19:616-28.

17. Kocar M, Telli F, Sonmez B, Kocar H. Adjuvant chemoradiotherapy combined with cisplatin, 5fluorouracil and folinic acid for locally advanced gastric cancer. J Oncol Sci. 2016;2:12-5. doi:10.1016/j.jons.2016.04.002.

18. Yalcin S. Gastric cancer in Turkey - A bridge between West and East. Gastrointest Cancer Res. 2009;3:29-32.

19. Ralf Hofheinz, Gerrit zur Hausen, Kersten Borchert, Albrecht Kretzschmar, Matthias Philip Ebert, Thomas Jens Ettrich, ... Michael Koenigsmann, Georg Martin Haag, Volker A. Hagen, Thomas Hoehler, Nils Homann, Hendrik Kroening, Peter Reichardt, Michael Rieg S-EA-B. Perioperative trastuzumab and pertuzumab in combination with FLOT versus FLOT alone for HER2 positive resectable esophagogastric adenocarcinoma: Petrarca-A phase II trial of the German AIO. J Clin Oncol. 2017;35 Suppl 15:TPS4133.

20. Tabernero J, Hoff PM, Shen L, Ohtsu A, Shah MA, Cheng K, et al. Pertuzumab plus trastuzumab and chemotherapy for HER2-positive metastatic gastric or gastro-oesophageal junction cancer (JACOB): final analysis of a double-blind, randomised, placebo-controlled phase 3 study. Lancet Oncol. 2018;19:1372-84. doi:10.1016/S1470-2045(18)30481-9.

\section{Tables}

Due to technical limitations, all tables are only available for download from the Supplementary Files section.

\section{Figures}


Figure 1

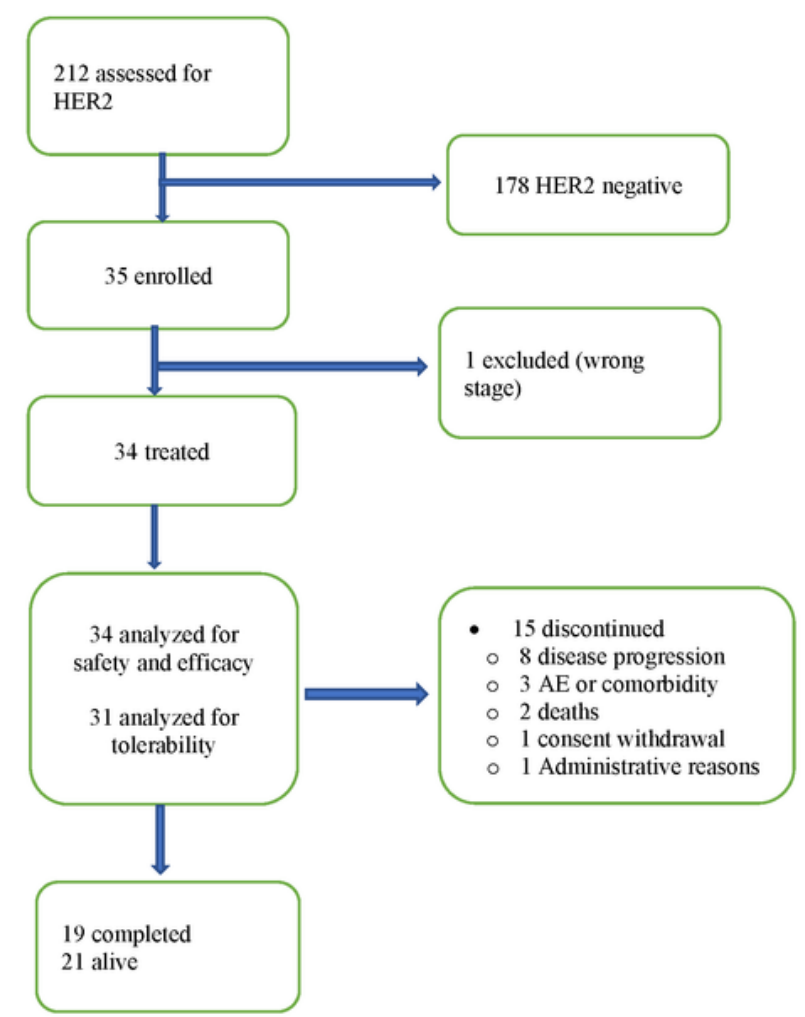

Figure 1

Consort Diagram 
Figure 2

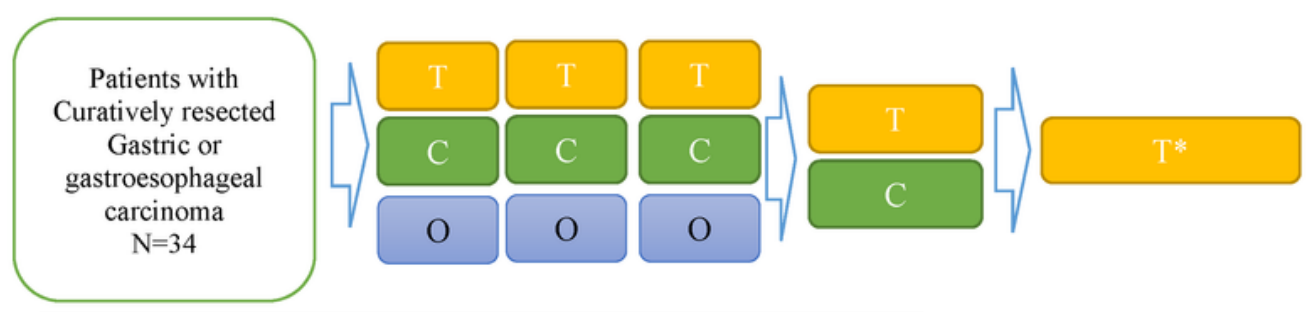

Open Label phase II trial

Primary end point: Tolerability and Safety

T: Trastuzumab, $\mathrm{T}^{*}$ : Trastuzumab maintanence $\mathrm{C}$ : Capecitabine, $\mathrm{O}$ :

Oxaliplatin

Figure 2

Treatment Schema 
Figure 3

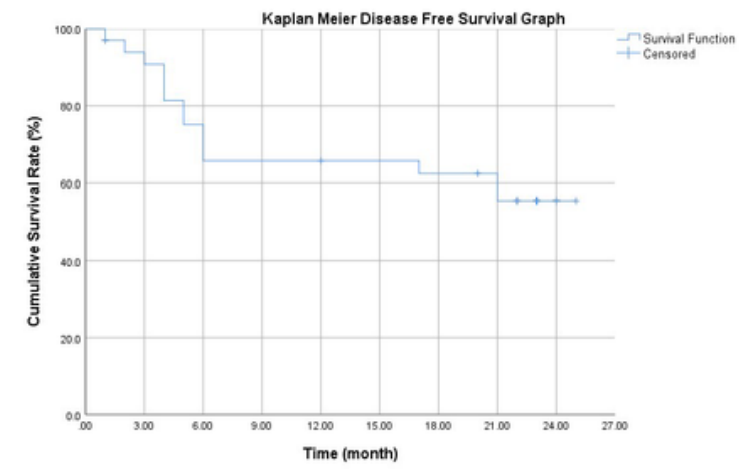

Figure 3

Disease-free survival curve for all patients 
Figure 4

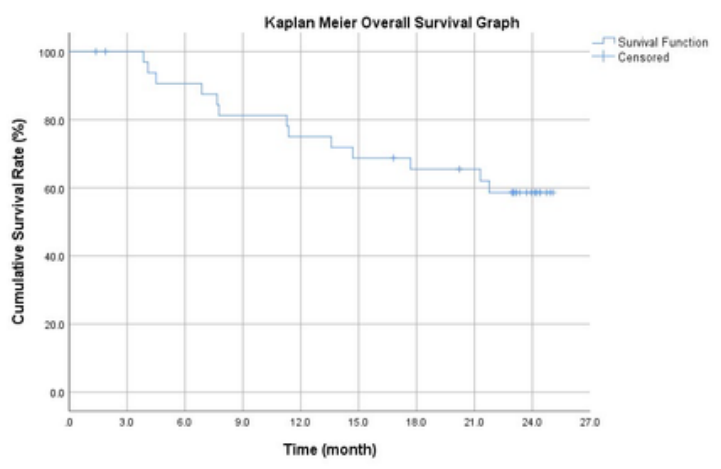

Figure 4

Overall survival curve for all patients

\section{Supplementary Files}

This is a list of supplementary files associated with this preprint. Click to download. 
- tables.pdf

Page 19/19 